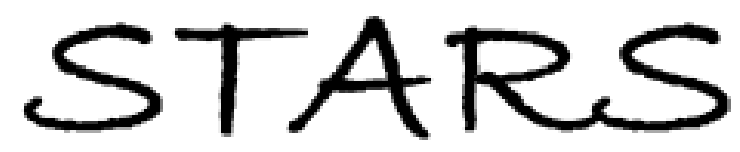

University of Central Florida

STARS

$1-1-2010$

\title{
Evidence of graphene-like electronic signature in silicene nanoribbons
}

\author{
Paola De Padova \\ Claudio Quaresima \\ Carlo Ottaviani \\ Polina M. Sheverdyaeva \\ Paolo Moras
}

See next page for additional authors

Find similar works at: https://stars.library.ucf.edu/facultybib2010

University of Central Florida Libraries http://library.ucf.edu

This Article is brought to you for free and open access by the Faculty Bibliography at STARS. It has been accepted for inclusion in Faculty Bibliography 2010 s by an authorized administrator of STARS. For more information, please contact STARS@ucf.edu.

\section{Recommended Citation}

Padova, Paola De; Quaresima, Claudio; Ottaviani, Carlo; Sheverdyaeva, Polina M.; Moras, Paolo; Carbone, Carlo; Topwal, Dinesh; Olivieri, Bruno; Kara, Abdelkader; Oughaddou, Hamid; Aufray, Bernard; and Lay, Guy Le, "Evidence of graphene-like electronic signature in silicene nanoribbons" (2010). Faculty Bibliography 2010s. 95.

https://stars.library.ucf.edu/facultybib2010/95

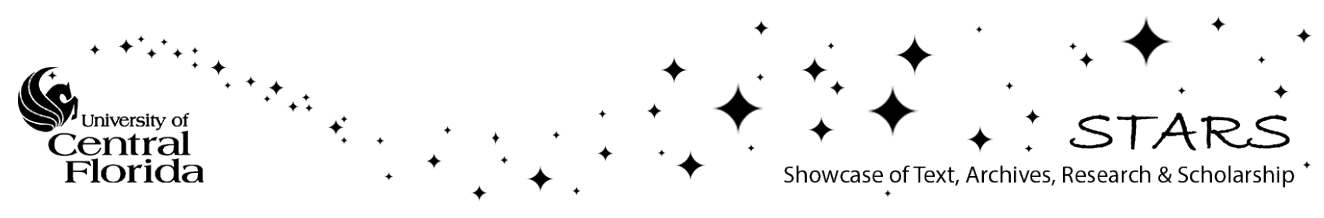




\section{Authors}

Paola De Padova, Claudio Quaresima, Carlo Ottaviani, Polina M. Sheverdyaeva, Paolo Moras, Carlo Carbone, Dinesh Topwal, Bruno Olivieri, Abdelkader Kara, Hamid Oughaddou, Bernard Aufray, and Guy Le Lay 


\section{Evidence of graphene-like electronic signature in silicene nanoribbons}

Cite as: Appl. Phys. Lett. 96, 261905 (2010); https://doi.org/10.1063/1.3459143

Submitted: 18 May 2010 . Accepted: 10 June 2010 . Published Online: 30 June 2010

Paola De Padova, Claudio Quaresima, Carlo Ottaviani, Polina M. Sheverdyaeva, Paolo Moras, Carlo Carbone, Dinesh Topwal, Bruno Olivieri, Abdelkader Kara, Hamid Oughaddou, Bernard Aufray, and Guy Le Lay

\section{ARTICLES YOU MAY BE INTERESTED IN}

Graphene-like silicon nanoribbons on $\mathrm{Ag}(110)$ : A possible formation of silicene Applied Physics Letters 96, 183102 (2010); https://doi.org/10.1063/1.3419932

Epitaxial growth of a silicene sheet

Applied Physics Letters 97, 223109 (2010); https://doi.org/10.1063/1.3524215

$s p^{2}$-like hybridization of silicon valence orbitals in silicene nanoribbons Applied Physics Letters 98, 081909 (2011); https://doi.org/10.1063/1.3557073

\section{Applied Physics Reviews} Now accepting original research 


\title{
Evidence of graphene-like electronic signature in silicene nanoribbons
}

\author{
Paola De Padova, ${ }^{1, a)}$ Claudio Quaresima, ${ }^{1}$ Carlo Ottaviani, ${ }^{1}$ Polina M. Sheverdyaeva, ${ }^{2}$ \\ Paolo Moras, ${ }^{3}$ Carlo Carbone, ${ }^{3}$ Dinesh Topwal, ${ }^{4}$ Bruno Olivieri, ${ }^{5}$ Abdelkader Kara, ${ }^{6}$ \\ Hamid Oughaddou, ${ }^{7}$ Bernard Aufray, ${ }^{8}$ and Guy Le Lay ${ }^{8, a)}$ \\ ${ }^{1}$ CNR-ISM, via Fosso del Cavaliere, 00133 Roma, Italy \\ ${ }^{2}$ Sincrotrone Trieste, S. S. Km163.5, I-34012 Basovizza (TS), Italy \\ ${ }^{3}$ CNR-ISM, Trieste, S. S. Km163.5, I-34012 Basovizza (TS), Italy \\ ${ }^{4}$ ICTP, Strada Costiera 11, 34151 Trieste, Italy \\ ${ }^{5}$ CNR-ISAC, via Fosso del Cavaliere, 00133 Roma, Italy \\ ${ }^{6}$ Department of Physics, University of Central Florida, Orlando, Florida 32816, USA \\ ${ }^{7}$ DSM/IRAMIS/SPCSI, CEA, Bât. 462, Saclay, 91191 Gif sur Yvette, France \\ ${ }^{8}$ CINaM-CNRS, Campus de Luminy, Case 913, 13288 Marseille Cedex 9, France
}

(Received 18 May 2010; accepted 10 June 2010; published online 30 June 2010)

\begin{abstract}
We report on the electronic properties of straight, $1.6 \mathrm{~nm}$ wide, silicene nanoribbons on $\operatorname{Ag}(110)$, arranged in a one-dimensional grating with a pitch of $2 \mathrm{~nm}$, whose high-resolution scanning tunneling microscopy images reveal a honeycomb geometry. Angle-resolved photoemission shows quantum confined electronic states of one-dimensional character. The silicon band dispersion along the direction of the nanoribbons suggests a behavior analogous to the Dirac cones of graphene on different substrates. () 2010 American Institute of Physics. [doi:10.1063/1.3459143]
\end{abstract}

The outstanding properties of graphene and the success in its preparation, from small flakes by mechanical exfoliation ${ }^{1}$ to large (centimeter in size) sheets upon deposition on polycrystalline copper foils, ${ }^{2}$ have generated a strong interest for its hypothetical silicon counterpart coined "silicene," that is, a one-atom thick silicon sheet arranged in a honeycomb lattice. ${ }^{3}$ Theory predicts free standing silicene sheets and ribbons to be stable either in a flat or in a low puckered configuration. In both cases they would present basically the same electronic properties of graphene. ${ }^{3-5}$ The predicted electronic structure of silicene presents $\pi$ and $\pi^{*}$ bands, which form so-called Dirac cones at the Brillouin zone (BZ) corners. Consequently, as in graphene, the charge carriers in silicene would behave as massless relativistic particles. Therefore, all graphene expectations, ${ }^{6}$ such as highspeed electronic nanometric devices based on ballistic transport at room temperature, could be transferred to this innovative material with the crucial advantage of easily fitting into the silicon-based electronics industry.

We have synthesized high aspect ratio silicon nanoribbons (SiNRs) upon in situ $\mathrm{Si}$ deposition onto silver (110) surfaces under ultrahigh vacuum conditions (we may suppose that other metals could eventually play a kind of similar catalytic role, provided they do not react with silicon to form silicides). ${ }^{7}$ These NRs are several nanometers in lengths, share a common width of $1.6 \mathrm{~nm}$ and are just $0.2 \mathrm{~nm}$ in height. They lay all parallel, in epitaxy, over the sample surface. At monolayer completion they form a grating with a pitch of just $2 \mathrm{~nm} .{ }^{8}$ High-resolution synchrotron radiation photoelectron spectroscopy measurements have revealed a strong metallic character of these SiNRs as well as the narrowest $\mathrm{Si} 2 p$ core-levels ever met in the solid state. ${ }^{9}$ We have recently shown through high-resolution scanning tunnel microscopy (STM) that these SiNRs display a honeycomb, silicene-like, arrangement. ${ }^{10,11}$ These observations confirm

\footnotetext{
a) Authors to whom correspondence should be addressed. Electronic addresses: depadova@ism.cnr.it and lelay@cinam.univ-mrs.fr.
}

the atomic structure of the SiNRs lying on the $\mathrm{Ag}(110)$ substrate predicted through density functional theory calculations in the general gradient approximation (DFT-GGA). Namely, these calculations yield a ground state geometry comprising a honeycomb silicon architecture arched on the substrate, indicating a silicene-like structure, mediated by the silver substrate. ${ }^{12}$

In the following we will describe angle-resolved photoelectron spectroscopy (ARPES) measurements of laterally compact SiNRs arranged in a one-dimensional (1D) grating. The electronic states deriving from the silicene structure display a quasi-1D character. We identify bands crossing the Fermi level, at the zone boundary, which are alike to the Dirac cones in graphene.

The STM images presented in the following have been recorded at the CINaM in Marseille (France). ARPES data have been measured at the VUV beamline of the synchrotron radiation facility ELETTRA in Trieste (Italy). The same procedure has been used for sample preparation and silicon deposition in both places. The $\operatorname{Ag}(110)$ substrate has been cleaned by Ar ion sputtering and annealing at $750 \mathrm{~K}$. Silicon has been evaporated at a rate of $\sim 0.03 \mathrm{ML} / \mathrm{min}$ from a Si source onto the Ag crystal kept at $\sim 440 \mathrm{~K}$. Under these conditions Si forms a 1D dense array, that is, a grating with a pitch of just $2 \mathrm{~nm}^{8}{ }^{8}$ This system displays a definite low energy electron diffraction pattern, with a sharp $5 \times 2 / 5 \times 4$ reconstruction (clear one-half order spots but comparatively weaker one-fourth order ones) ${ }^{8}$

Figure 1(a) displays a $11 \times 10 \mathrm{~nm}^{2}$ filled-states STM image of the SiNRs on the $\operatorname{Ag}(110)$ surface. This image shows dense SiNRs with a common width of $1.6 \mathrm{~nm}$, equal to $4 \mathrm{a}_{\mathrm{Ag}[100]}$, where $\mathrm{a}_{\mathrm{Ag}[100]}=0.409 \mathrm{~nm}$, aligned along the $\mathrm{Ag}$ $[-110]$ direction. $^{8}$ The silicene-like atomic structure observed in STM images is supported by DFT calculations in the GGA approximation. ${ }^{10-12}$ Figure 1(b) shows the ball model of the calculated atomic structure of the dense array of SiNRs. 


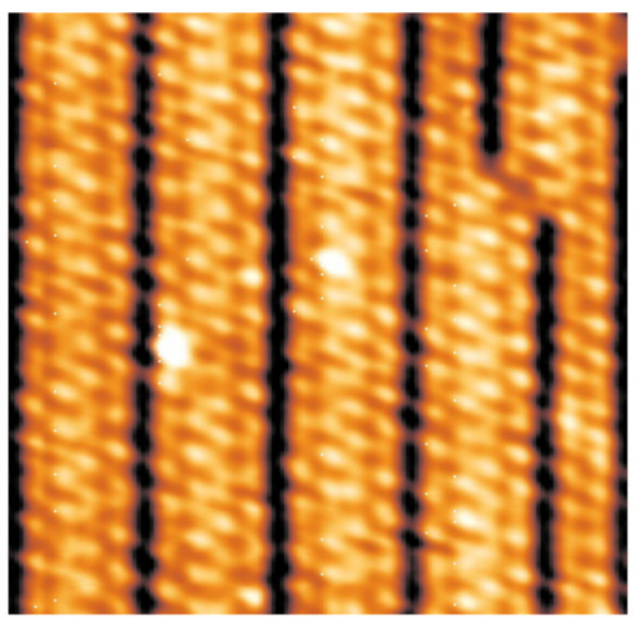

(a)
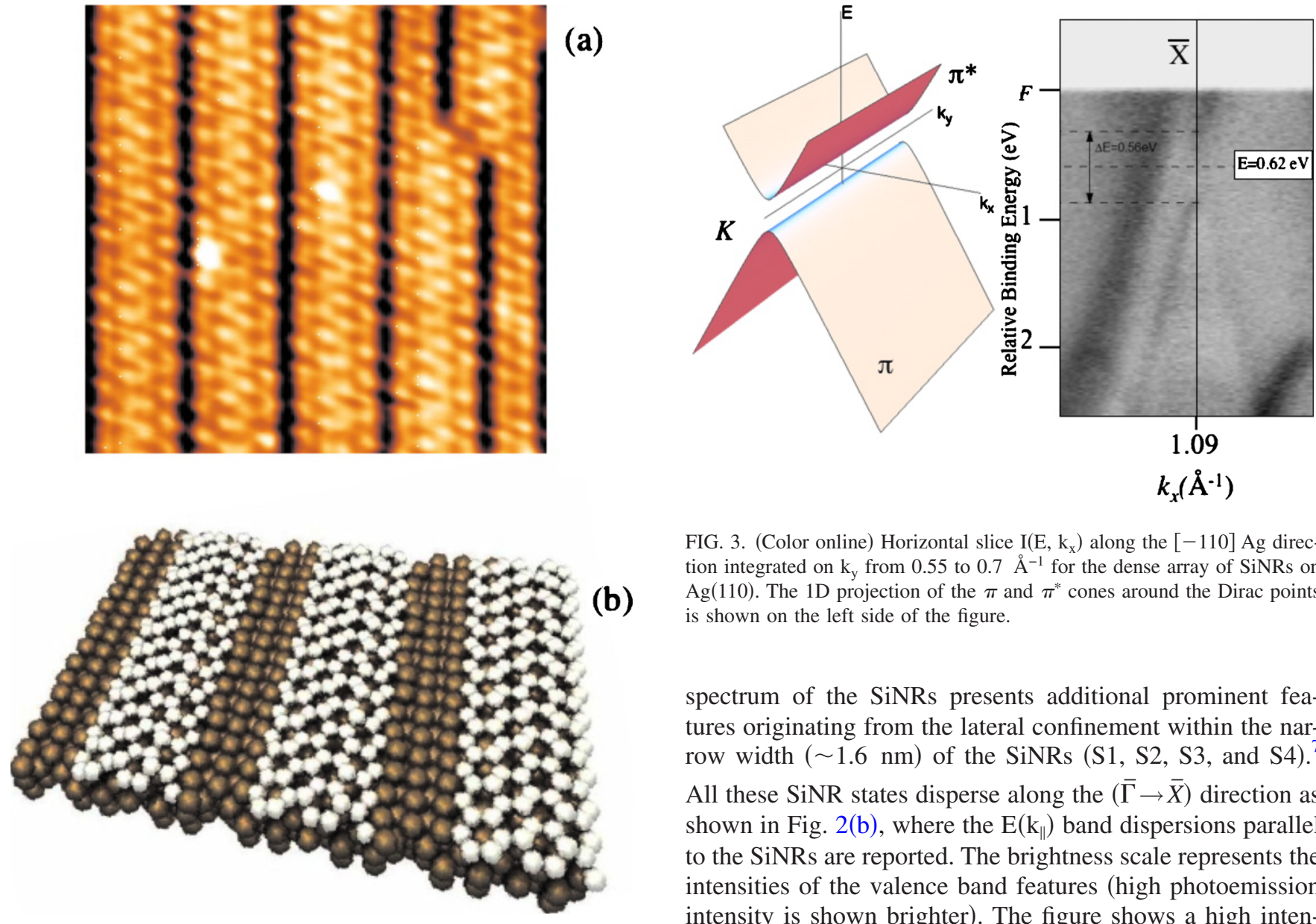

FIG. 1. (Color online) Filled-states STM image, $11 \times 10 \mathrm{~nm}^{2}(\mathrm{~V}=-3.3 \mathrm{~V}$, $\mathrm{I}=1.90 \mathrm{nA})$ of the dense array of SiNRs forming a 1D grating with a pitch of $\sim 2 \mathrm{~nm}$ (a); ball model of the corresponding calculated atomic structure [see Ref. 12] (b).

We will describe now ARPES measurements of the valence states associated to the SiNR array. Figure 2(a) shows the electrons energy distribution curves at the $\bar{X}$ point of the Ag surface Brillouin zone. On the bare $\operatorname{Ag}(110)$ the spectrum in the vicinity of $\mathrm{E}_{\mathrm{F}}$ is dominated by the $\mathrm{Ag} s p$ band. The

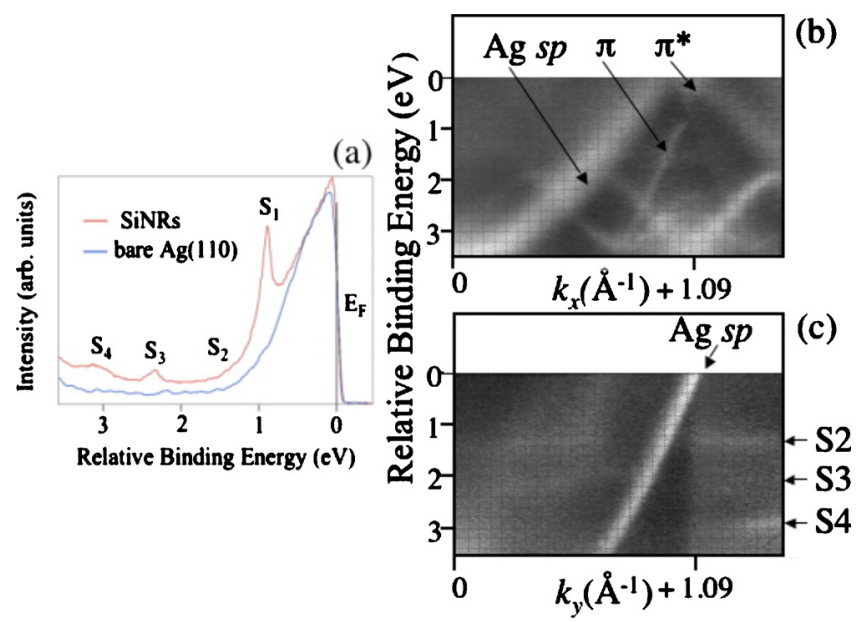

FIG. 2. (Color online) Energy distribution curves for bare $\operatorname{Ag}(110)$ and for the array of SiNRs (a); band dispersion for the array of SiNRs vs $\mathrm{k}_{\mathrm{x}}$ (along the SiNRs) at $\mathrm{k}_{\mathrm{y}}=0.7 \AA^{-1}$ (b) and vs $\mathrm{k}_{\mathrm{y}}$ (perpendicular to the SiNRs) at $\mathrm{k}_{\mathrm{x}}=0.35 \AA^{-1}$ (c). The photon energy used was $\mathrm{h} \nu=78 \mathrm{eV}$.
FIG. 3. (Color online) Horizontal slice $\mathrm{I}\left(\mathrm{E}, \mathrm{k}_{\mathrm{x}}\right)$ along the $[-110] \mathrm{Ag}$ direction integrated on $\mathrm{k}_{\mathrm{y}}$ from 0.55 to $0.7 \AA^{-1}$ for the dense array of SiNRs on $\mathrm{Ag}(110)$. The 1D projection of the $\pi$ and $\pi^{*}$ cones around the Dirac points

spectrum of the SiNRs presents additional prominent features originating from the lateral confinement within the narrow width $(\sim 1.6 \mathrm{~nm})$ of the SiNRs $\left(\mathrm{S} 1, \mathrm{~S} 2, \mathrm{~S} 3\right.$, and S4). ${ }^{7}$ All these SiNR states disperse along the $(\bar{\Gamma} \rightarrow \bar{X})$ direction as shown in Fig. 2(b), where the $\mathrm{E}\left(\mathrm{k}_{\|}\right)$band dispersions parallel to the SiNRs are reported. The brightness scale represents the intensities of the valence band features (high photoemission intensity is shown brighter). The figure shows a high intensity Ag $s p$ band and several SiNRs bands dispersing versus $\mathrm{k}_{\mathrm{x}}$ (along the SiNRs) at $\mathrm{k}_{\mathrm{y}}=0.7 \AA^{-1}$ (perpendicular to the SiNRs). The $1 \mathrm{D}$ character $^{7}$ of these quantum states is testified by the dispersionless band behavior along the direction orthogonal to the SiNRs (the Ag [001] direction) [see Fig. 2(c)]. Interestingly, one notices in Fig. 2(b) practically linear dispersions of the silicon bands near the $\bar{X}$ point of the $\mathrm{Ag}$ surface BZ $\left(\mathrm{k}_{\mathrm{x}}= \pm 1.09 \AA^{-1}\right)$.

In order to better describe the behavior of the silicene bands, Fig. 3 displays the photoemission data for $\mathrm{k}_{\mathrm{x}}$ in the vicinity of the $\bar{X}$ point and for $\mathrm{k}_{\mathrm{y}}$ integrated from 0.55 to $0.70 \AA^{-1}$. In this momentum range there is little overlap with the more intense Ag bands. One can observe two branches of highly dispersive $\mathrm{Si}$ bands separated by a gap of about $0.5 \mathrm{eV}$ and centered at $0.6 \mathrm{eV}$ below $\mathrm{E}_{\mathrm{F}}$. This is reminiscent of the $\pi^{*}$ (upper branch) and $\pi$ (lower branch) bands of silicene predicted by theory, ${ }^{5}$ although shifted below $E_{F}$ and separated by a gap. We attribute this downward shift and the opening of the gap to the interaction with the $\operatorname{Ag}(110)$ substrate. Typically, this behavior is very similar to that of the $\pi^{*}$ and $\pi$ band dispersions near the K point of graphene grown on different surfaces, where charge transfer pulls down the $\pi^{*}$ branch and the interaction with the substrate opens a gap. ${ }^{13,14}$

Following this analogy, we identify the observed bands as the $\pi^{*}$ (upper branch) and the $\pi$ (lower branch) of our SiNRs. This interpretation is consistent with the STM images showing that the internal hexagonal atomic structure of the NRs is oriented as in zig-zag cut honeycomb sheets with apexes aligned along their lengths. In this case the projected $\mathrm{K}$ point of the silicene Brillouin zone coincides with the $\bar{X}$ is shown on the left side of the figure. 
point of the Ag surface Brillouin zone. The normally empty $\pi^{*}$ branch is populated due to charge transfer from the substrate to the SiNRs in accord with the Miedema electronegativities (Ag 4.45, Si 4.70). ${ }^{15}$ The opening of the gap may be related to the arched honeycomb architecture of the SiNRs, anchored along their sides to the Ag substrate, as revealed in STM images ${ }^{10}$ and in line with the DFT-GGA calculations. ${ }^{12}$ The coincidence of the $\bar{X}$ point in $\mathrm{Ag}$ and $\mathrm{K}$ point in silicene yields a $\mathrm{Si}-\mathrm{Si}$ nearest neighbor distance within the hexagons of the honeycomb silicene lattice of $2.24 \AA$, in excellent accord with the mean $\mathrm{Si}-\mathrm{Si}$ distance within the honeycomblike structure arched on the substrate derived from the DFTGGA calculations. ${ }^{12,16}$

Presently, calculations of the band dispersions for silicene NRs only exist for free standing ones, ${ }^{5}$ which makes direct comparison with our experimental results somewhat risky. However, we can note that spin-polarized metallic zigzag NRs result from energy minimization, ${ }^{5}$ which is in line with our measurements and calculations.

To conclude, we have investigated by angle-resolved photoemission the electronic structure of a grating of SiNRs grown on the $\operatorname{Ag}(110)$ surface. The SiNRs appear to be a 1D honeycomb material, whose growth is mediated by the anisotropic silver substrate. The dispersion of the bands allows us to identify states corresponding to the 1D projection of $\pi$ and $\pi^{*}$ Dirac cones in silicene. Their location in the reciprocal space confirms the structural parameters derived from the STM and theoretical analysis.

The authors thank the International Collaboration between CNR and CNRS 2008-2009 through the project "Self- assembled silicon nanowires: tailoring of their structural, electronic and magnetic properties." We acknowledge financial support through the EUROCORES SANMAG project of the European Science Foundation (Contract No. ERAS-CT2003-980409).

${ }^{1}$ K. S. Novoselov, A. K. Geim, S. V. Morozov, D. Jiang, Y. Zhang, S. V. Dubonos, I. V. Grigorieva, and A. Firsov, Science 306, 666 (2004).

${ }^{2}$ X. Li, W. Cai, J. An, S. Kim, J. Nah, D. Yang, R. Piner, A. Velamkanni, I. Jung, E. Tutuc, S. K. Banerjee, L. Colombo, and R. S. Ruoff, Science 324, 1312 (2009).

${ }^{3}$ G. G. Guzmán-Verri and L. C. Lew Yan Voon, Phys. Rev. B 76, 075131 (2007).

${ }^{4}$ S. Lebègue and O. Eriksson, Phys. Rev. B 79, 115409 (2009).

${ }^{5}$ S. Cahangirov, M. Topsakal, E. Aktuerk, H. Sahin, and S. Ciraci, Phys. Rev. Lett. 102, 236804 (2009)

${ }^{6}$ A. K. Geim and K. S. Novoselov, Nature Mater. 6, 183 (2007).

${ }^{7}$ C. Léandri, G. Le Lay, B. Aufray, C. Girardeaux, J. Avila, M. E. Davila, M. C. Asensio, C. Ottaviani, and A. Cricenti, Surf. Sci. 574, L9 (2005).

${ }^{8}$ H. Sahaf, L. Masson, C. Léandri, B. Aufray, G. Le Lay, and F. Ronci, Appl. Phys. Lett. 90, 263110 (2007).

${ }^{9}$ P. De Padova, C. Quaresima, P. Perfetti, B. Olivieri, C. Léandri, B. Aufray, S. Vizzini, and G. Le Lay, Nano Lett. 8, 271 (2008).

${ }^{10}$ B. Aufray, A. Kara, S. Vizzini, H. Oughaddou, C. Léandri, B. Ealet, and G. Le Lay, Appl. Phys. Lett. 96, 183102 (2010).

${ }^{11}$ G. Le Lay, B. Aufray, C. Léandri, H. Oughaddou, J. P. Biberian, P. De Padova, M. E. Davila, B. Ealet, and A. Kara, Appl. Surf. Sci. 256, 524 (2009).

${ }^{12}$ A. Kara, S. Vizzini, C. Léandri, B. Ealet, H. Oughaddou, B. Aufray, and G. Le Lay, J. Phys.: Condens. Matter 22, 045004 (2010).

${ }^{13}$ J. Wintterlin and M.-L. Bocquet, Surf. Sci. 603, 1841 (2009).

${ }^{14}$ A. Grüneis, K. Kummer, and D. V. Vyalikh, New J. Phys. 11, 073050 (2009).

${ }^{15}$ W. Mönch, Phys. Rev. B 37, 7129 (1988).

${ }^{16}$ A. Kara, C. Léandri, M. E. Davila, P. De Padova, B. Ealet, B. Aufray, and G. Le Lay, J. Supercond. Novel Magn. 22, 259 (2009). 